\title{
Frequent CCNE1 amplification in endometrial intraepithelial carcinoma and uterine serous carcinoma
}

\author{
Elisabetta Kuhn ${ }^{1}$, Asli Bahadirli-Talbott ${ }^{1}$ and Ie-Ming Shih ${ }^{1,2}$ \\ ${ }^{1}$ Department of Pathology, Baltimore, MD, USA and ${ }^{2}$ Departments of Gynecology and Obstetrics, and \\ Oncology, The Johns Hopkins Medical Institutions, Baltimore, MD, USA
}

\begin{abstract}
Uterine serous carcinoma accounts for only $10 \%$ of all uterine epithelial cancers, but is the leading cause of death among them. The pathogenesis of this aggressive neoplasm has been largely elusive until recently, when comprehensive genome-wide analyses of uterine serous carcinoma have been performed. Among amplified cancer-related genes, CCNE1, encoding for cyclin E1, is frequently amplified in uterine serous carcinoma. In the current study we applied fluorescence in situ hybridization (FISH) to determine CCNE1 copy number in uterine serous carcinoma and concurrent endometrial intraepithelial carcinoma, the noninvasive component of uterine serous carcinoma, and the results were correlated with clinicopathological and molecular features. We found that $20(45 \%)$ of 44 uterine serous carcinomas and $11(41 \%)$ of 27 endometrial intraepithelial carcinomas showed CCNE1 amplification. Overall, we found high concordance in CCNE1 copy number in concurrent uterine serous carcinoma and endometrial intraepithelial carcinoma pairs $(P$-value $=0.0003)$. No correlation was observed between CCNE1 copy number and clinicopathological features, as well as common mutations previously reported in uterine serous carcinoma. In summary, we confirm that amplification of CCNE1 is a frequent molecular genetic change in uterine serous carcinoma. Moreover, the identification of CCNE1 amplification in many endometrial intraepithelial carcinomas suggests that this genetic event occurs early during tumor progression.

Modern Pathology (2014) 27, 1014-1019; doi:10.1038/modpathol.2013.209; published online 6 December 2013
\end{abstract}

Keywords: amplification; CCNE1; cyclin E1; endometrial intraepithelial carcinoma; FISH; fluorescence in situ hybridization; serous carcinoma

Uterine serous carcinoma accounts for only $10 \%$ of all uterine corpus epithelial cancers, but is the leading cause of endometrial cancer-related death.1,2 The pathogenesis of this aggressive neoplasm has been largely elusive until recently, when comprehensive genome-wide analyses of uterine serous carcinoma were performed. ${ }^{3-6}$ Whole-exome sequencing revealed that TP53, PIK3CA, FBXW7 and PPP2R1A are the most commonly mutated genes in uterine serous carcinoma. ${ }^{3}$ Furthermore, we applied SNP arrays for copy number analysis in 23 uterine serous carcinomas, and identified high levels of amplification of CCNE1, encoding for cyclin E1, in $26.1 \%$ of the cases. ${ }^{3}$ This result was subsequently validated by other investigators

Correspondence: Dr E Kuhn, MD and Professor I-M Shih, MD, PhD, Department of Pathology, Johns Hopkins University, 1550 Orleans Street, CRBII, room 376, Baltimore, MD 21231, USA. E-mail: elisabettakuhn@hotmail.it and ishih@jhmi.edu

Received 2 September 2013; accepted 26 September 2013; published online 6 December 2013 including those from The Cancer Genome Atlas consortium. ${ }^{4,5}$

Cyclin E1 is a nuclear protein critically involved in the regulation of cell cycle by promoting the transition from G1 phase to $S$ phase. ${ }^{7}$ In normal cells, cyclin E1 is expressed when the cells undergo DNA synthesis, and once the task is completed, cyclin E1 expression decreases. One of the mechanisms responsible for cyclin E1 degradation is mediated by ubiquitination through the SCF-Fbxw7 protein complex. ${ }^{8}$ Aberrant accumulation of cyclin E1 is common in a variety of carcinomas of breast, cervix, colorectum, stomach, and ovary, ${ }^{9,10}$ and as a result, a high level of cyclin E1 shortenings G1 phase, expedites G1/S transition and facilitates cellular proliferation. Interestingly, somatic mutations of FBXW7, a gene encoding Fbxw7, are frequently mutated in uterine serous carcinomas including those uterine serous carcinomas without CCNE1 amplification. ${ }^{3-5}$ Defective Fbxw7 is unable to promote cyclin E1 degradation and, consequently, mutated Fbxw7 indirectly enhances cyclin E1 expression. 
Thus, approximately half of uterine serous carcinomas had either a molecular genetic alteration in FBXW7 or CCNE1 amplification that may contribute to cyclin E1 overexpression. ${ }^{3,4}$ These observations underscore the role of cyclin E-FBXW7 pathway in the development of uterine serous carcinoma.

In the current study, we applied fluorescence in situ hybridization (FISH) to determine CCNE1 copy number at a single-cell resolution in uterine serous carcinoma and concurrent endometrial intraepithelial carcinoma. We also correlated the findings with other molecular alterations and clinicopathological features. Our results not only confirm the frequent amplification of CCNE1 in uterine serous carcinoma but also provide new evidence that increased CCNE1 copy number has occurred in the noninvasive stage of uterine serous carcinoma progression, suggesting that CCNE1 amplification represents one of the early molecular events in the pathogenesis of uterine serous carcinoma.

\section{Materials and methods}

\section{Case Selection}

A total of 44 uterine serous carcinomas and 27 concurrent endometrial intraepithelial carcinomas were retrieved from the archival file at the Department of Pathology, the Johns Hopkins Hospital (Baltimore, MD). All the available slides were reviewed to confirm the diagnosis by two pathologists (EK and IMS). Both clinicopathological features and molecular characteristics were recorded. ${ }^{3}$ This study was approved by the Institutional Review Board of the Johns Hopkins Medical Institutions.

\section{Fluorescence In Situ Hybridization}

Two-color FISH assay was used to measure the gene copy number of CCNE1 in endometrial intraepithelial carcinoma and uterine serous carcinoma. Briefly, 4- $\mu \mathrm{m}$ thick sections were incubated at $62{ }^{\circ} \mathrm{C}$ for 30 min, deparaffinized in xylene, hydrated through graded ethanol, and placed in deionized water. The sections were incubated in Trilogy ${ }^{\mathrm{TM}}$ (cat\# CMX833, Cell Marque, Austin, TX), at $88^{\circ} \mathrm{C}$ for $40 \mathrm{~min}$ and then washed in $2 \mathrm{X}$ Aniara saline-sodium citrate (SSC). The slides were then placed in a denaturation solution (70\% formamide/2X SCC) at $75^{\circ} \mathrm{C}$ for $5 \mathrm{~min}$, rinsed in 2X SSC and allowed to cool down to room temperature for $5 \mathrm{~min}$. The slides were then dehydrated through graded ethanol, and dried in an oven at $62{ }^{\circ} \mathrm{C}$ for 2 min. FISH probes for CCNE1 and CEP19, the centromeric region of chromosome 19 (cat\# FG0013, Abnova Corp, Taipei, Taiwan) were applied to the slides according to the vendor's instructions. Denaturation was accomplished by incubating the slides at $80{ }^{\circ} \mathrm{C}$ for $15 \mathrm{~min}$. Hybridization was performed at $37^{\circ} \mathrm{C}$ for $20-24 \mathrm{~h}$. The slides were washed in $1.5 \mathrm{~mol} / \mathrm{l}$ urea in $0.2 \mathrm{X}$ SSC for $20 \mathrm{~min}$, followed by washing in
2X SSC at room temperature for $2 \mathrm{~min}$. The slides were drained, dehydrated through graded ethanol, air-dried, mounted with ProLong Gold Antifade Reagent with DAPI (cat\# P-36931, Invitrogen, Eugene, Oregon, USA) and imaged. ${ }^{11,12}$

Three images from each lesion were acquired at 400X magnification using a Nikon 50i epifluorescence microscope equipped with fluorescence excitation/emission filters for different fluorophores (Omega Optical). Grayscale images were captured using the Nikon NIS-Elements software and an attached Photometrics CoolSNAP EZ digital camera. For presentation and measurement purposes, images were pseudo-colored and merged. We analyzed the CCNE1 copy number per cell in at least 50 non-overlapping nuclei. The CCNE1 gene copy number was classified into five FISH strata as previously described. ${ }^{13}$ Specifically we defined: (1) CCNE1 amplification as the presence of either loose or tight CCNE1 cluster, or the ratio of CCNE1 to centromeric probe (CEP19) $\geq 2$ in more than $20 \%$ of the analyzed tumor cells, (2) high polisomy with $\geq 4$ copies in $\geq 40 \%$ cells, (3) low polisomy with $\geq 4$ copies in 10-40\% cells, (4) trisomy with $\geq 3$ copies in $\geq 10 \%$ cells and $\geq 4$ copies in $<10 \%$ cells, (5) disomy with $3-4$ copies in $<10 \%$ cells. CCNE1 amplification was considered to have increased gene copy number (FISH positive), and the categories from disomy to low polysomy were considered not to have increased gene copy number (FISH negative).

\section{Statistical Analysis}

Comparisons of FISH outcomes between matched endometrial intraepithelial carcinoma and uterine serous carcinoma were obtained using the one-tailed Wilcoxon signed-rank test. A simple linear regression model was used to determine the relationship of the ratio of CCNE1/CEP19 FISH signals between uterine serous carcinoma and endometrial intraepithelial carcinoma. $R^{2}$ value was calculated. Comparisons of FISH outcome with clinicopathological and molecular data were performed using the two-tailed unpaired Fisher's exact test. Kaplan-Meier survival plot was generated to compare overall survival between patients with different CCNE1 FISH status, and difference between survival curves were estimated using the long-rank test. $P$-values of 0.05 or less were considered statistically significant. Statistical analysis was carried out using the GraphPad Prism software version 5.0 (GraphPad Software, San Diego, CA, USA).

\section{Results}

CCNE1 Copy Number Analysis in Concurrent Uterine Serous Carcinoma and Endometrial Intraepithelial Carcinoma

Gene copy number of CCNE1 was determined in 44 uterine serous carcinomas and 27 concurrent 
endometrial intraepithelial carcinomas using twocolor FISH assay. Overall, the frequency of copy number gain in CCNE1 (FISH positive: amplification; Figures 1 and 2) was similar between uterine serous carcinoma (20 of $44,45 \%$ ) and endometrial intraepithelial carcinoma (11 of 27, 41\%) (Table 1). Notably, the level of CCNE1 copy number gain in amplified samples was remarkably high (CCNE1) CEP19 ratio 1.3-13.2, median 3.8, Figure 2).

The concordance of CCNE1 copy number abnormalities between uterine serous carcinoma and endometrial intraepithelial carcinoma from the same case was high (25 of $27,93 \% ; P$-value $=$ 0.0003). Fifteen (56\%) of 25 matched uterine serous carcinoma and endometrial intraepithelial carcinoma pairs were FISH negative in both lesions, whereas $10(37 \%)$ matched lesions showed CCNE1 amplification in both tumor lesions (Table 2). Discordance in CCNE1 copy number status was detected in only two $(7 \%)$ cases; one of these uterine serous carcinomas had a gain in copy number while endometrial intraepithelial carcinoma was FISH negative, and vice versa in the remaining case. Linear regression also showed a significant correlation of CCNE1/CEP19 ratio between uterine serous carcinoma and endometrial intraepithelial carcinoma with a $R^{2}$ of 0.900 (Figure 3).

We also observed that three $(7 \%)$ uterine serous carcinomas showed intratumoral heterogeneity as manifested by regional difference in CCNE1 copy number alteration, because there were tumor areas with focal CCNE1 amplification that were adjacent to tumor cells without amplification.

\section{CCNE1 Copy Number and Somatic Gene Mutations}

Based on exome sequencing, we have recently reported TP53, FBXW7, PIK3CA, and PPP2R1A as the most commonly mutated genes in uterine serous carcinoma. ${ }^{3}$ Information regarding the presence of somatic mutations of these four genes was available in 40 cases. We identified TP53, FBXW7, PIK3CA, and PPP2R1A somatic mutations in 32 (80\%), 11 (28\%), 6 $(15 \%)$, and $6(15 \%)$ of 40 cases, respectively. ${ }^{3}$ There was no correlation between the presence of any of these mutations and CCNE1 gene amplification $(P$-values $>0.05$, Table 3$)$.

\section{CCNE1 Copy Number and Clinicopathological Characteristics}

Clinical information was available in 44 cases. No evidence of correlation was observed between CCNE1 copy number and clinicopathological features, including age, race, clinical, and pathological stage, overall survival, and angiolymphatic invasion.

\section{Discussion}

Recent DNA copy number analyses using SNP arrays have demonstrated that CCNE1 is one of the most commonly amplified genes in uterine serous carcinoma as it occurs in $23-48 \%$ of uterine serous carcinomas. $^{3-5}$ However, the prevalence of CCNE1 amplification has not been reported at the tissue level and it is unclear if CCNE1 amplification occurs during early stages of tumor development. In the current study we performed two-color FISH assay on 44 uterine serous carcinomas and detected CCNE1 amplification in $45 \%$ of them. Furthermore, we also found that CCNE1 was amplified in endometrial intraepithelial carcinoma, the noninvasive component of uterine serous carcinoma, indicating that DNA copy number gain of CCNE1 has occurred at early stages of tumor development. The above results should have several biological and clinical implications.

Like ovarian high-grade serous carcinoma, uterine serous carcinoma is characterized by frequent CCNE1 amplification in addition to TP53 mutations in the majority of cases. ${ }^{3,4}$ Because both uterine serous carcinoma and ovarian high-grade serous carcinoma develop along the type II pathway in endometrial and ovarian cancers, respectively, this finding raises a possibility that both TP53 mutation and CCNE1 amplification are the defining features of type II tumors arising from gynecological organs. In fact, based on The Cancer Genome Atlas endome-
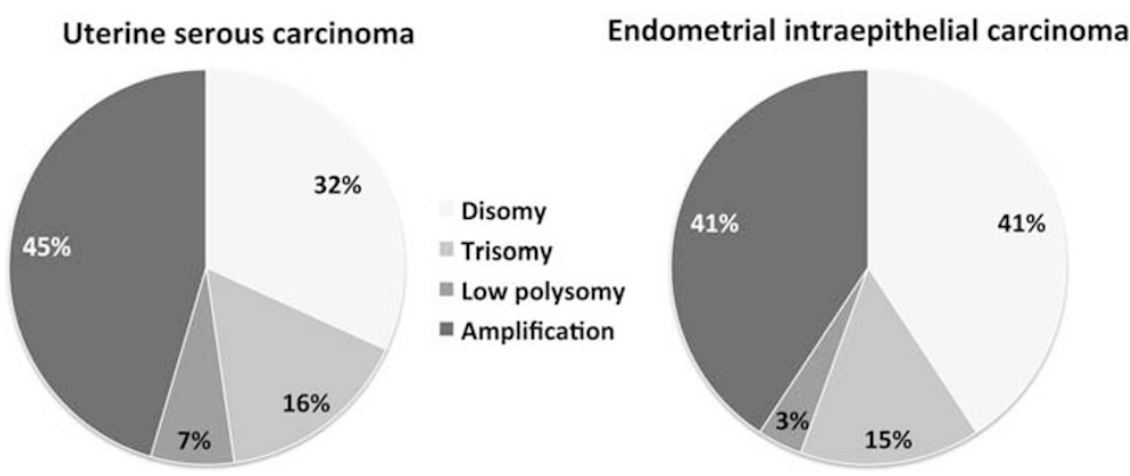

Figure 1 Summary of percentage of cases showing different CCNE1 copy number changes. A total of 44 primary uterine serous carcinomas and 27 endometrial intraepithelial carcinomas were analyzed by fluorescence in situ hybridization. 

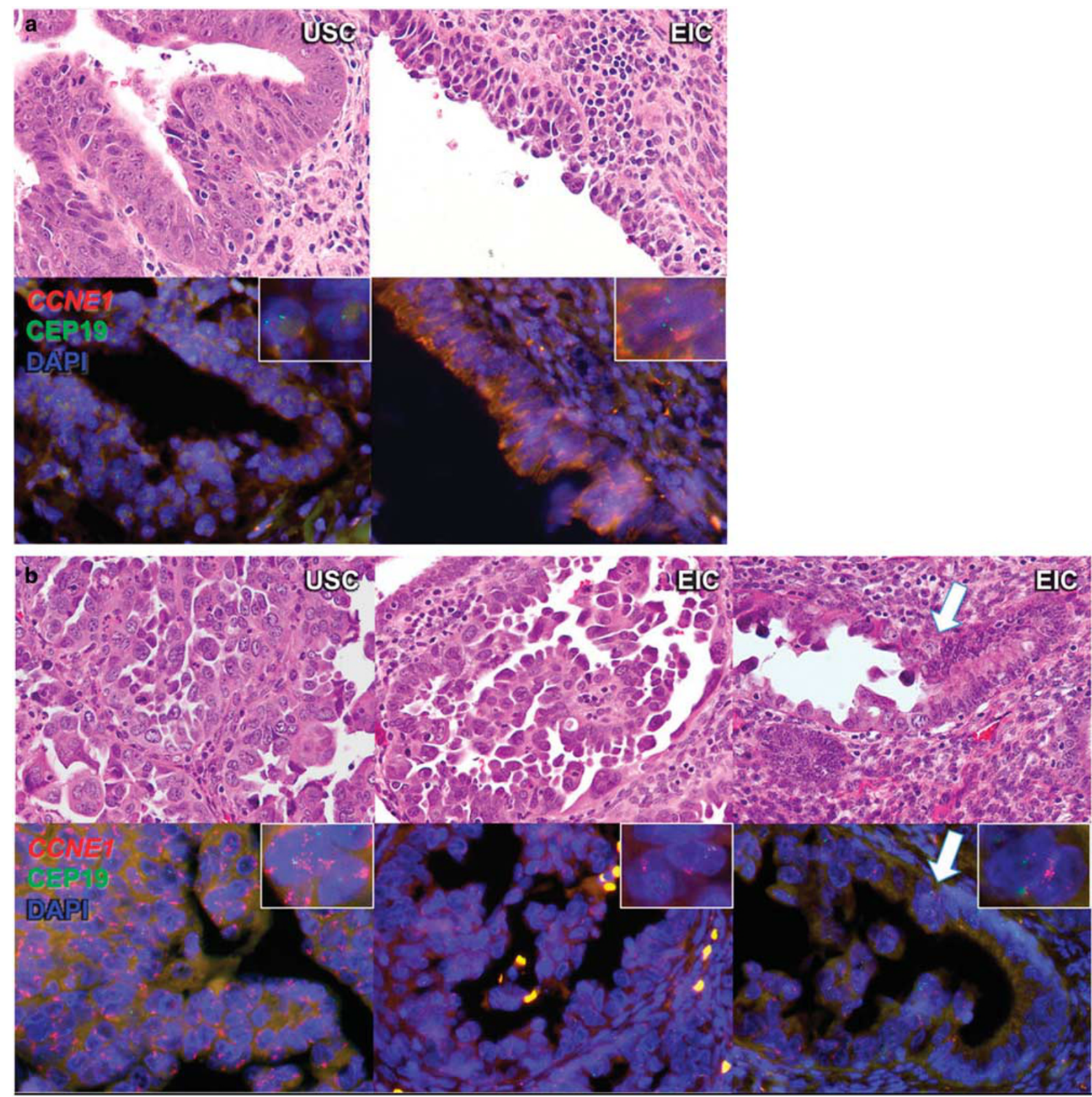

Figure 2 Examples of uterine serous carcinoma (USC) and concurrent endometrial intraepithelial carcinoma (EIC) (hematoxylin-eosin staining, top panels) with corresponding CCNE1 status by fluorescence in situ hybridization (FISH) (bottom panels). (a) Both cancer components present disomy for CCNE1 (red) as compared with centromeric probe, CEP19 (green). (b) FISH demonstrates CCNE1 amplification in both uterine serous carcinoma and endometrial intraepithelial carcinoma components in another case. The arrow indicates the junction between normal-appearing endometrial epithelium and CCNE1-amplified endometrial intraepithelial carcinoma. Inserts: higher magnification.

trial cancer data set, CCNE1 amplification and TP53 mutations are specifically identified in 'serous-like' carcinomas $(P$-value $<0.0001) .^{5}$ As compared with type I neoplasms, type II carcinomas are highly aggressive with worse overall survival. Previous studies have shown that type II cancers, including uterine serous carcinoma and ovarian high-grade serous carcinoma, are characterized by prominent
DNA copy number alterations, which reflect a history of chromosomal instability. ${ }^{3}$ Interestingly, CCNE1 amplification and TP53 mutations have been known to participate in promoting chromosomal instability, a feature essential for tumor development in many types of human cancer. ${ }^{14-17}$

In addition to the roles in chromosomal instability, cyclin E1 upregulation is critical for cellular 
Table 1 CCNE1 copy number by fluorescence in situ hybridization (FISH) in 44 primary uterine serous carcinomas and 27 endometrial intraepithelial carcinomas

\begin{tabular}{lcc}
\hline $\begin{array}{l}\text { Copy number } \\
\text { category }\end{array}$ & $\begin{array}{c}\text { Endometrial } \\
\text { Carcinoma, } \mathrm{n}(\%)\end{array}$ & $\begin{array}{c}\text { Entraepithelial } \\
\text { carcinoma, } \mathrm{n}(\%)\end{array}$ \\
\hline FISH negative & $24(55)$ & $16(59)$ \\
$\quad$ Disomy & $14(32)$ & $11(41)$ \\
Trisomy & $7(16)$ & $4(15)$ \\
Low polysomy & $3(7)$ & $1(3)$ \\
FISH Positive & $20(45)$ & $11(41)$ \\
Amplification & $20(45)$ & $11(41)$ \\
Total & $44(100)$ & $27(100)$ \\
\hline
\end{tabular}

Abbreviation: n, number of cases.

Table 2 Correlation between CCNE1 copy number by fluorescence in situ hybridization (FISH) in 27 primary uterine serous carcinomas and concurrent endometrial intraepithelial carcinomas

\begin{tabular}{lcc}
\hline $\begin{array}{l}\text { Copy number } \\
\text { category }\end{array}$ & $\begin{array}{c}\text { Uterine serous } \\
\text { carcinoma, n (\%) }\end{array}$ & $\begin{array}{c}\text { Endometrial } \\
\text { intraepithelial } \\
\text { carcinoma, n (\%) }\end{array}$ \\
\hline $\begin{array}{l}\text { FISH negative } \\
\text { Disomy }\end{array}$ & $16(59)$ & $16(59)$ \\
Trisomy & $10(37)$ & $11(41)$ \\
Low polysomy & $4(15)$ & $4(15)$ \\
FISH Positive & $2(7)$ & $1(3)$ \\
Amplification & $11(41)$ & $11(41)$ \\
Total & $11(41)$ & $27(100)$ \\
\hline
\end{tabular}

Abbreviation: n, number of cases.

proliferation. For example, in breast and ovarian cancers, in vitro silencing of CCNE1 suppresses cellular growth selectively in cells harboring CCNE1 amplification. ${ }^{10,18,19}$ On the other hand, ectopic expression of CCNE1 increases cellular proliferation in ovarian cancer cell lines. ${ }^{18}$ Moreover, these data support the driver role of CCNE1 amplification in cancers as cyclin E1 mediates G1/S transition through the activation of CDK2. Interestingly, the survival of breast cancer cells harboring CCNE1 gene amplification depends on CDK2 expression and kinase activity. ${ }^{18}$ Consequently, CCNE1 amplification is a potential biomarker of sensitivity to CDK2 inhibitors.

Like ovarian high-grade serous carcinomas of which many may develop from serous tubal intraepithelial carcinoma, uterine serous carcinoma frequently co-exists with endometrial intraepithelial carcinoma, the noninvasive component, which has been thought as a precursor lesion of uterine serous carcinoma. $^{3,20,21}$ CCNE1 amplification in many endometrial intraepithelial carcinomas suggests that this genetic event occurs early during tumor progression of uterine serous carcinoma. Thus, both TP53 mutation and CCNE1 amplification are associated with early tumor development, as in ovarian

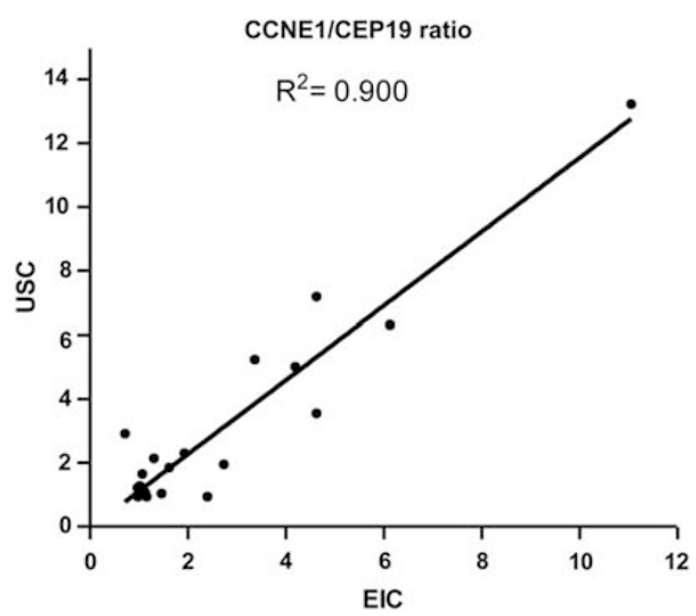

Figure 3 Correlation of CCNE1 copy number between uterine serous carcinoma (USC) and endometrial intraepithelial carcinoma (EIC) from the same patients. The CCNE1 copy number was presented as the ratio of CCNE1 to CEP19, the centromeric region, and linear regression was used to model the relationship of the CCNE1 copy number between uterine serous carcinoma and endometrial intraepithelial carcinoma. Each dot represents an individual case.

Table 3 Correlation between CCNE1 amplification by fluorescence in situ hybridization in 40 primary uterine serous carcinomas and somatic mutations in FBXW7, PIK3CA, PPP2R1A and TP53

\begin{tabular}{lccccc}
\hline & & $\begin{array}{c}\text { FBXW7 } \\
\text { Tutation, } \\
\mathrm{n}(\%)^{\mathrm{a}}\end{array}$ & $\begin{array}{c}\text { PIK3CA } \\
\text { mutation, } \\
\mathrm{n}(\%)^{\mathrm{a}}\end{array}$ & $\begin{array}{c}\text { PPP2R1A } \\
\text { mutation, } \\
\mathrm{n}(\%)^{\mathrm{a}}\end{array}$ & $\begin{array}{c}\text { TP53 } \\
\text { mutation, } \\
\mathrm{n}(\%)^{\mathrm{a}}\end{array}$ \\
\hline $\begin{array}{l}\text { TCNE1 } \\
\text { amplified }\end{array}$ & 17 & $6(35)$ & $2(12)$ & $3(18)$ & $15(88)$ \\
$\begin{array}{l}C C N E 1 \\
\text { nonamplified } \\
\text { Total }\end{array}$ & 23 & $5(22)$ & $4(17)$ & $3(13)$ & $17(74)$ \\
& 40 & $11(28)$ & $6(15)$ & $6(15)$ & $32(80)$
\end{tabular}

Abbreviation: $\mathrm{n}$, number of cases mutated.

aPercentage of mutations in CCNE1 amplified, non-amplified and total sample groups.

high-grade serous carcinoma. ${ }^{20,22}$ Since TP53 mutation and cyclin E1 upregulation are cardinal features of endometrial intraepithelial carcinoma, it would be of great interest to determine if both molecular changes lead to tumorigenesis in endometrial epithelial cells in animal models.

Finally, the lack of an association between CCNE1 amplification and clinical features may indicate that CCNE1 amplification is important during very early stages of tumor development and thus does not impact on the aggressive behavior of uterine serous carcinoma. Similarly, the findings from The Cancer Genome Atlas did not show an association between CCNE1 amplification and overall survival in endometrial serous-like carcinomas. ${ }^{5}$ Alternatively, it may be due to a limited number of cases analyzed in this study. 
In conclusion, our FISH analysis provides cogent evidence that CCNE1 amplification is a common molecular genetic alteration in uterine serous carcinoma and endometrial intraepithelial carcinoma. This finding validates the previous observation from genome-wide analysis based on comprehensive DNA copy number changes. Further studies are required to better delineate the clinical and biological impact of CCNE1 amplification on overall survival and therapy response in uterine serous carcinoma patients.

\section{Disclosure/conflict of interest}

The authors declare no conflict of interest.

\section{References}

1 Lee KR, Tavassoli FA, Prat J, et al. Tumors of the ovary and peritoneum. In: Tavassoli FA, Devilee P (eds). World Health Organization Tumours of the Breast and Female Genital System. IARC Press: Lyon, France, 2003, pp 224-225.

2 Hamilton CA, Cheung MK, Osann K, et al. Uterine papillary serous and clear cell carcinomas predict for poorer survival compared to grade 3 endometrioid corpus cancers. Br J Cancer 2006;94:642-646.

3 Kuhn E, Wu RC, Guan B, et al. Genome-wide analyses of uterine serous carcinoma identify pathway aberrations involving cyclinE-Fbxw7, PI3K and p53. J Natl Cancer Inst 2012;104:1503-1513.

4 Zhao S, Choi M, Overton JD, et al. Landscape of somatic single-nucleotide and copy-number mutations in uterine serous carcinoma. Proc Natl Acad Sci USA 2013;110:2916-2921.

5 Cancer Genome Atlas Research NetworkKandoth C, Schultz N, et al. Integrated genomic characterization of endometrial carcinoma. Nature 2013;497:67-73.

6 Le Gallo M, O’Hara AJ, Rudd ML, et al. Exome sequencing of serous endometrial tumors identifies recurrent somatic mutations in chromatin-remodeling and ubiquitin ligase complex genes. Nat Genet 2012;44:1310-1315.

7 Geisen C, Moroy T. The oncogenic activity of cyclin E is not confined to Cdk2 activation alone but relies on several other, distinct functions of the protein. J Biol Chem 2002;277:39909-39918.

8 Onoyama I, Nakayama KI. Fbxw7 in cell cycle exit and stem cell maintenance: insight from gene-targeted mice. Cell Cycle 2008;7:3307-3313.
9 Donnellan R, Chetty R. Cyclin E in human cancers. FASEB J 1999;13:773-780.

10 Natrajan R, Mackay A, Wilkerson PM, et al. Functional characterization of the 19q12 amplicon in grade III breast cancers. Breast Cancer Res 2012;14:R53.

11 Kuhn E, Ayhan A, Shih IeM, et al. The pathogenesis of atypical proliferative Brenner tumor: an immunohistochemical and molecular genetic analysis. Mod Pathol; advance online publication, 26 July 2013; doi:10.1038/modpathol.2013.142 (e-pub ahead of print).

12 Kuhn E, Meeker AK, Visvanathan K, et al. Telomere length in different histologic types of ovarian carcinoma with emphasis on clear cell carcinoma. Mod Pathol 2011;24:1139-1145.

13 Varella-Garcia M. Stratification of non-small cell lung cancer patients for therapy with epidermal growth factor receptor inhibitors: the EGFR fluorescence in situ hybridization assay. Diagn Pathol 2006;1:19.

14 Loeb KR, Kostner H, Firpo E, et al. A mouse model for cyclin E-dependent genetic instability and tumorigenesis. Cancer Cell 2005;8:35-47.

15 Spruck CH, Won KA, Reed SI. Deregulated cyclin E induces chromosome instability. Nature 1999;401: 297-300.

16 Duensing A, Duensing S. Guilt by association? p53 and the development of aneuploidy in cancer. Biochem Biophys Res Commun 2005;331:694-700.

17 Song H, Hollstein M, Xu Y. p53 gain-of-function cancer mutants induce genetic instability by inactivating ATM. Nat Cell Biol 2007;9:573-580.

18 Nakayama N, Nakayama K, Shamima Y, et al. Gene amplification CCNE1 is related to poor survival and potential therapeutic target in ovarian cancer. Cancer 2010;116:2621-2634.

19 Etemadmoghadam D, George J, Cowin PA, et al. Amplicon-dependent CCNE1 expression is critical for clonogenic survival after cisplatin treatment and is correlated with 20q11 gain in ovarian cancer. PLoS One 2010;5:e15498.

20 Kuhn E, Kurman RJ, Vang R, et al. TP53 mutations in serous tubal intraepithelial carcinoma and concurrent pelvic high-grade serous carcinoma-evidence supporting the clonal relationship of the two lesions. J Pathol 2012;226:421-426.

21 Kuhn E, Meeker A, Wang TL, et al. Shortened telomeres in serous tubal intraepithelial carcinoma: an early event in ovarian high-grade serous carcinogenesis. Am J Surg Pathol 2010;34:829-836.

22 Kuhn E, Bahadirli-Talbott A, Kurman R, et al. CCNE1 amplification may precede centrosome number abnormality in progression from serous tubal intraepithelial carcinoma to high-grade serous carcinomã. Mod Pathol 2013;26:283A. 